\title{
Monte-Carlo dosimetry on a realistic cell monolayer geometry exposed to alpha particles
}

\author{
Ph Barberet F Vianna M Karamitros T Brun N Gordillo \\ Ph Moretto S Incerti and $H$ Seznec
}

\begin{abstract}
The energy and specific energy absorbed in the main cell compartments (nucleus and cytoplasm) in typical radiobiology experiments are usually estimated by calculations as they are not accessible for a direct measurement. In most of the work, the cell geometry is modelled using the combination of simple mathematical volumes. We propose a method based on high resolution confocal imaging and ion beam analysis (IBA) in order to import realistic cell nuclei geometries in Monte-Carlo simulations and thus take into account the variety of different geometries encountered in a typical cell population. Seventy-six cell nuclei have been imaged using confocal microscopy and their chemical composition has been measured using IBA. A cellular phantom was created from these data using the ImageJ image analysis software and imported in the Geant4 Monte-Carlo simulation toolkit. Total energy and specific energy distributions in the 76 cell nuclei have been calculated for two types of irradiation protocols: a $3 \mathrm{MeV}$ alpha particle microbeam used for targeted irradiation and a ${ }^{239} \mathrm{Pu}$ alpha source used for large angle random irradiation. Qualitative images of the energy deposited along the particle tracks have been produced and show good agreement with images of DNA double strand break signalling proteins obtained experimentally. The methodology presented in this paper provides microdosimetric quantities calculated from realistic cellular volumes. It is based on open-source oriented software that is publicly available.
\end{abstract}

(Some figures may appear in colour only in the online journal)

\section{Introduction}

Understanding the biological effects of ionizing radiation at the cellular scale remains one of the challenges of today's radiobiology research. To address this question, experimental 
irradiation facilities have been developed worldwide to expose living cells in vitro to different types of ionizing radiation ( $\mathrm{x}$ - or gamma rays, electrons, ions and neutrons). Among the different types of ionizing radiation, ions are the ones presenting the higher linear energy transfer and thus creating the denser molecular damages in cells. Understanding the effects of such radiation on living cells is of primary importance considering their presence in the telluric radioactivity (alpha particles) and in cosmic rays, as well as their increasing use in medical applications (proton and hadron therapy, alpha particle immunotherapy) (Fokas et al 2009, Dadachova 2010, Mulford et al 2005). Irradiation facilities allowing the exposure of living cells to ions in order to study specific biological responses to a given exposure are usually based on radioactive sources (alpha particle emitting isotopes), broad beams or microbeams delivered by accelerators. Nevertheless, the physical interactions of the charged particles inside the cells cannot usually be measured directly since they take place in a micrometre scale volume immersed in nutrient medium. For this reason, more and more refined simulation tools have been developed to model the energy deposition track-structure patterns and the cellular response (Nikjoo et al 1998, 2006).

In this work, two irradiation facilities delivering light ions in the $\mathrm{MeV}$ energy range have been developed: a microbeam used for targeted irradiation with $3 \mathrm{MeV}$ alpha particles or protons (Barberet et al 2005 ) and a ${ }^{239} \mathrm{Pu}$ alpha source used for large angle random irradiation. In addition, computational methods based on the Geant4 simulation toolkit (Agostinelli et al 2003, Apostolakis et al 2009) have been developed to model in detail these irradiation facilities as well as the geometry of the cell (Incerti et al 2009). This led to a code that fully models the microbeam irradiation of a single cell that is now available for download in the Geant4 toolkit as an advanced example (the so-called 'microbeam' advanced example) that is regularly updated and improved, following the toolkit bi-annual releases. The motivation of this work was to investigate the dependence of the specific energy (energy absorbed per mass unit as defined in ICRU report 60 (ICRU 1998), expressed in Gray) absorbed in the main cell compartments (nucleus and cytoplasm) with the cell morphology and composition. Indeed, as the specific energy is not accessible to a direct measurement, it is usually calculated by assuming simple cell geometries based on the combination of simple mathematical volumes (spheres, ellipsoids, cylinders, etc) (Alard et al 2002, Beaton et al 2011, Champion et al 2008, Roeske et al 2007,2008, Tung et al 2004). These approximations do not take into account the variety of different geometries encountered in a typical cell population (e.g. cell-cycle status). The method developed by Incerti et al was based on the modelling of single cell geometry (nucleus and cytoplasm) obtained from high resolution confocal microscopy and its chemical composition was measured experimentally by ion beam analysis (IBA) at the micrometre scale. This approach allowed us to obtain the specific energy for a $3 \mathrm{MeV}$ incoming alpha particle and to underline the specific energy dependence with the cell morphology. However, the main limitation was the possibility to model only one cell as the cellular phantom was built from a high resolution image taken from a single cell. More recently, authors have reported the interest of using confocal microscopy to model 3D multi-cellular tissues in dosimetry simulations associated with electron microbeam irradiation (Miller et al 2011). In this study the confocal images were used to quantify the physical features of skin model tissues required for track-structure simulations but, to our knowledge, they were not introduced in a Monte-Carlo code to create a cellular/tissular phantom. In this work, we report an extension of the approach initially applied to a single cell to a cell monolayer (76 cells) allowing thus to take into account the variety of cell geometries encountered in a typical population. Total absorbed energy and specific energy distributions obtained by simulating two irradiation devices are shown as well as qualitative images of the energy deposited along the ion tracks. The dependence of the specific energy on the nuclear density is also discussed. 


\section{Materials and methods}

\subsection{Cell irradiation and cell imaging}

2.1.1. HaCaT and HaCaT/(H2BGFP)Tg cell lines. HaCaT cells (Boukamp et al 1988) can easily be plated as a monolayer with a homogeneous cellular and nuclear thickness, so that a fairly uniform exposure could be obtained. HaCaT cells were maintained in culture during $24 \mathrm{~h}$ in Dulbecco's Modified Eagle Medium (DMEM, Invitrogen) supplemented with $10 \%$ foetal calf serum (Invitrogen), $2.5 \mathrm{mM} \mathrm{L}$-glutamine (Invitrogen) and $100 \mu \mathrm{g} \mathrm{ml}{ }^{-1}$ streptomycin/penicillin (Invitrogen) at $37{ }^{\circ} \mathrm{C}$ in a $95 \%$ air $/ 5 \% \mathrm{CO}_{2}$ and $\mathrm{H}_{2} \mathrm{O}$ saturated atmosphere.

Exponentially growing $\mathrm{HaCaT}$ cells were stably transfected with H2B-GFP expression vector (Kanda et al 1998) using a Lipofectamin transfection kit (Invitrogen) according to the manufacturer's protocol and stably transfected clones were obtained using $500 \mu \mathrm{g} \mathrm{ml}^{-1} \mathrm{G} 418$ selections (Invitrogen). The selected transfected $\mathrm{HaCaT}$ cells, expressing the recombinant histone H2B-GFP, were named 'HaCaT/(GFP-H2B)Tg'.

2.1.2. Cell preparation for alpha particle irradiation. Polyethylene cylindrical dishes have been designed to keep the cells fully immersed in their culture medium during the irradiation procedure. The cells attach on $3.8 \mu \mathrm{m}$ thick polypropylene foil (Goodfellow) coated with CellTak $\left(2.54 \mu \mathrm{g} \mathrm{cm}^{-2}\right.$ ) (BD-Biosciences) to enhance cell attachment. This foil is stretched to close the entrance face of the dish and allows the traversal of the incoming alpha particles; the back side is sealed by a $200 \mu \mathrm{m}$ thick circular glass microscope slide squeezed on a silicon ring joint by means of screws. $24 \mathrm{~h}$ prior to irradiation, HaCaT cells were platted on the $3.8 \mu \mathrm{m}$ thick polypropylene foil and were grown in DMEM (Invitrogen) supplemented with $10 \%$ foetal calf serum (Invitrogen), $2.5 \mathrm{mM} \mathrm{L-glutamine} \mathrm{(Invitrogen)} \mathrm{and} 100 \mu \mathrm{g} \mathrm{ml}^{-1}$ streptomycin/penicillin (Invitrogen) at $37^{\circ} \mathrm{C}$ in a $95 \%$ air $/ 5 \% \mathrm{CO}_{2}$ and $\mathrm{H}_{2} \mathrm{O}$-saturated atmosphere.

2.1.3. Microbeam irradiation. Targeted irradiation is performed with the microbeam irradiation facility developed at the CENBG (Centre d'Etudes Nucléaires de BordeauxGradignan, France). During the irradiation procedure, the cell dish is positioned vertically by a high precision stage situated between the beam extraction window and the epifluorescence microscope coupled to a high resolution CCD camera. Each CCD frame is immediately transmitted to a personal computer where every cell is recognized automatically using image processing routines (Image Pro Plus, Media Cybernetics) implemented in the software designed to control the overall experiment (Barberet et al 2005). An automated irradiation procedure, based on a mosaic of microscope fields that covers the entire culture dish, has been designed. The location of the individual nuclei is determined by imaging the fluorescent staining pattern of the fluorescent histone H2BGFP. When a target position is reached, a predefined number of particles are delivered in the cell and the automatic procedure continues with the next target position. The energy of the incident alpha particles delivered by the accelerator is $3 \mathrm{MeV}$ and $2.34 \mathrm{MeV}$ at the cell-polypropylene interface.

After irradiation the cells are maintained in culture during $2 \mathrm{~h}$ in DMEM (Invitrogen) supplemented with $10 \%$ foetal calf serum (Invitrogen), $2.5 \mathrm{mM} \mathrm{L-glutamine} \mathrm{(Invitrogen)} \mathrm{and}$ $100 \mu \mathrm{g} \mathrm{ml}{ }^{-1}$ streptomycin/penicillin (Sigma) at $37^{\circ} \mathrm{C}$ in a $95 \%$ air $/ 5 \% \mathrm{CO}_{2}$ and $\mathrm{H}_{2} \mathrm{O}-$ saturated atmosphere. Then, the medium is removed and the cells are treated according to the immuno-labelling protocol. 


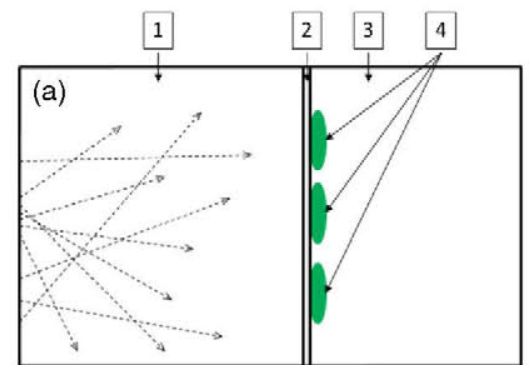

1: $1 \mathrm{~mm}$ thick air layer

2: $4 \mu \mathrm{m}$ thick polypropylene

3: Growth medium (water)

4: Cellular phantom

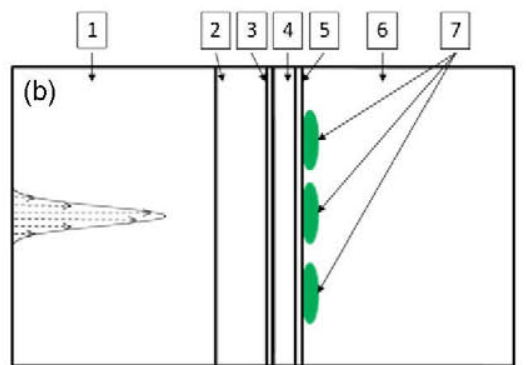

1: Vacuum

2: $4 \mathrm{~mm}$ thick isobutane layer (10 mbar)

3: $150 \mathrm{~nm}$ thick $\mathrm{Si}_{3} \mathrm{~N}_{4}$ window

4: $200 \mu \mathrm{m}$ thick air layer

5: $4 \mu \mathrm{m}$ thick polypropylene

6: Growth medium (water)

7: Cellular phantom

Figure 1. Scheme of the two irradiation facilities used. (a) ${ }^{239} \mathrm{Pu}$ source irradiation set-up and (b) microbeam irradiation facility. The microbeam is modelled as a Gaussian beam presenting a $1 \mu \mathrm{m}$ FWHM under vacuum. The dashed arrows symbolize the distribution of incoming particles.

2.1.4. ${ }^{239} \mathrm{Pu}$ irradiation. $\quad$ A second charged particle irradiation device, based on a $3.7 \mathrm{kBq}$ ${ }^{239} \mathrm{Pu}$ source, has been developed for experiments that do not require a precise targeting of individual cells and for quick protocol testing. This set-up was designed to allow the use of the same cell dish as the one developed for the microbeam irradiation facility. The alpha particles (5105 keV: $12 \%$; $5143 \mathrm{keV}: 15 \%$; $5156 \mathrm{keV}: 73 \%$ ) randomly emitted by the ${ }^{239} \mathrm{Pu}$ nuclei traverse $1 \mathrm{~mm}$ of air prior to reaching the $3.8 \mu \mathrm{m}$ thick polypropylene support on which the cells attach. The source active area is $1.5 \mathrm{~cm}$ in diameter. The layout of the radioactive source irradiation set-up is shown in figure 1.

Compared to other alpha particle irradiation devices presented in the literature (Szabó et al 2002, Tisnek et al 2009, Søyland and Hasfjell 2000), the set-up used in this work is based on a rather long radioactive period isotope $\left({ }^{239} \mathrm{Pu}\right.$, period: 24110 years). This isotope presents the advantage of being an almost pure alpha emitter and that the source can be used over long periods without a significant decrease of its activity. Moreover, the long radioactive period of ${ }^{239} \mathrm{Pu}$ avoids the presence of a significant amount of its progeny in the source.

Alpha particle irradiators are usually designed to carry out radiobiology measurements such as cell survival or relative biological effectiveness calculations, etc. These measurements require the use of relatively high doses. The set-up developed in this work has been designed to obtain a few traversals per nucleus for a few minutes' irradiation, mainly for DNA damage and repair studies. The activity of the alpha particle source was thus chosen relatively low ( $\mathrm{kBq}$ instead of $\mathrm{MBq}$ or $\mathrm{GBq}$ in other studies).

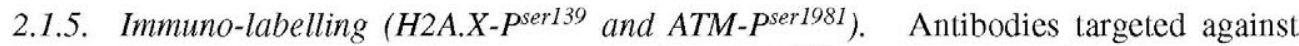
DNA double strand break signalling, such as H2A.X-P ${ }^{\text {ser139 }}$ (also called $\gamma$-H2A.X) and ATM$\mathrm{P}^{\text {ser1981 }}$, were used to visualize radiation-induced DNA damages. Radiation-induced DNA double strand breaks can be easily visualized by the presence of discrete foci corresponding to the accumulation of DNA signalling proteins. HaCaT cells grown during $2 \mathrm{~h}$ post-irradiation on the $3.8 \mu \mathrm{m}$ thick polypropylene film were washed with phosphate buffer saline (PBS1X, $\mathrm{pH} 7.5$ ) and fixed for $15 \mathrm{~min}$ at room temperature (RT) in $1 \%(\mathrm{w} / \mathrm{v})$ paraformaldehyde and 
treated with $50 \mathrm{mM} \mathrm{NH} 4 \mathrm{Cl}, 15 \mathrm{~min}$ at RT. Cells were washed and stored in $70 \%$ ethanol at $4{ }^{\circ} \mathrm{C}$. Then, they were re-hydrated by incubation in PBS1X for $10 \mathrm{~min}$ at RT, and were subsequently incubated with the primary antibody, directed against H2A.X-Pser139 ${ }^{\text {or ATM- }}$ $\mathrm{P}^{s e r} 1981$ in PBS1X $+2 \%(\mathrm{w} / \mathrm{v})$ bovine serum albumine $(\mathrm{BSA})+0.05 \%(\mathrm{v} / \mathrm{v})$ Triton X-100 overnight at $4{ }^{\circ} \mathrm{C}$. Cells were washed three times in PBS1X $+2 \%(\mathrm{w} / \mathrm{v}) \mathrm{BSA}+0.05 \%(\mathrm{v} / \mathrm{v})$ Triton X-100, for 15 min each, and then incubated with the secondary antibody (AlexaFluor594 Goat-anti-Rabbit or AlexaFluor594 Goat-anti-Mouse, Molecular Probes) at $4{ }^{\circ} \mathrm{C}$ overnight. Hoecsht ${ }^{33342}$ counter-staining is finally used as a control to reveal all cell nuclei. The samples were embedded with ProLong Gold Antifade Reagent (Molecular Probes), mounted and stored in the dark at $-20^{\circ} \mathrm{C}$.

2.1.6. Image acquisition with confocal laser scanning microscopy: geometry of the cellular phantom. Three-dimensional images were acquired with a Leica DMRE TCS SP2 AOBS confocal microscope (oil-immersion objective of $\times 40,1.25$ numerical aperture), assembled and reconstructed using the Leica Confocal ${ }^{\mathrm{TM}}$ software. During the $z$-acquisition, data were recorded from the GFP signal and stacks of horizontal slices were collected automatically. The image resolution was set to $0.732 \mu \mathrm{m}$ in the horizontal plane and to $0.529 \mu \mathrm{m}$ in the $z$ direction. Sets of 50 to 100 slices were recorded per $z$-stack to map the entire nuclei present in the field of view. Image acquisition was performed using an 8 bit, $512 \times 512$ pixel resolution in horizontal slices. A field of view was chosen to obtain a reasonable number of cells in the image (about 100 cells). Image stacks were stored as Tiff files and transferred to a PC running on Microsoft Windows XP ${ }^{\mathrm{TM}}$.

2.1.7. Cell preparation for ion beam microanalysis. Cells were cultured directly onto ion beam microprobe sample holders (Simon et al 2011). Briefly, cells were directly grown at $60 \%$ confluence on $2 \mu \mathrm{m}$ thick polycarbonate foil for $24 \mathrm{~h}$ in DMEM (Invitrogen) supplemented with $10 \%$ foetal calf serum (Invitrogen), $2.5 \mathrm{mM}$-glutamine (Invitrogen) and $100 \mu \mathrm{g} \mathrm{ml} \mathrm{m}^{-1}$ streptomycin/penicillin (Sigma) at $37{ }^{\circ} \mathrm{C}$ in a $95 \%$ air $/ 5 \% \mathrm{CO}_{2}$ and $\mathrm{H}_{2} \mathrm{O}$ saturated atmosphere. Cells were rinsed twice in DMEM, rinsed in PBS1X and very briefly rinsed in ultrapure water to remove excess of extracellular salts from PBS1X. Finally, cells were cryofixed at $-160^{\circ} \mathrm{C}$ into liquid nitrogen chilled isopentane, and freeze-dried at $-35^{\circ} \mathrm{C}$ for $96 \mathrm{~h}$. The cryofixation followed by freeze-drying has been shown to preserve the sample elemental distribution (Carmona et al 2008).

2.1.8. Ion beam analysis (IBA): chemical composition of the cellular phantom. The cell nuclei constituting the phantom were filled with a chemical composition measured using IBA. For this purpose, quantitative chemical element imaging was carried out using the high resolution beam line of the AIFIRA (Applications Interdisciplinaires des Faisceaux d'Ions en Région Aquitaine) facility using three complementary ion beam analytical techniques: PIXE (particle induced x-ray emission), RBS (Rutherford backscattering spectroscopy) and STIM (scanning transmission ion microscopy) (Simon et al 2011, Barberet et al 2011). Freeze-dried cells were analysed using a $1.5 \mathrm{MeV}$ proton microbeam (focused down to a diameter of about $1 \mu \mathrm{m}$ for PIXE and RBS and about $300 \mathrm{~nm}$ for STIM). Measurements on narrow areas containing a limited number of cells (usually about ten) were carried out. The ion beam lateral resolution enabled to discriminate the cytoplasmic and nuclear areas of the cells as shown in figure 2. Quantitative chemical composition of low- $Z$ major elements $(C, N$ and $O$ ) was obtained from RBS analysis and trace element composition ( $\mathrm{P}, \mathrm{S}, \mathrm{K}$ ) was obtained from PIXE. Concentrations in hydrated samples (see table 1) were calculated by assuming that the cell 

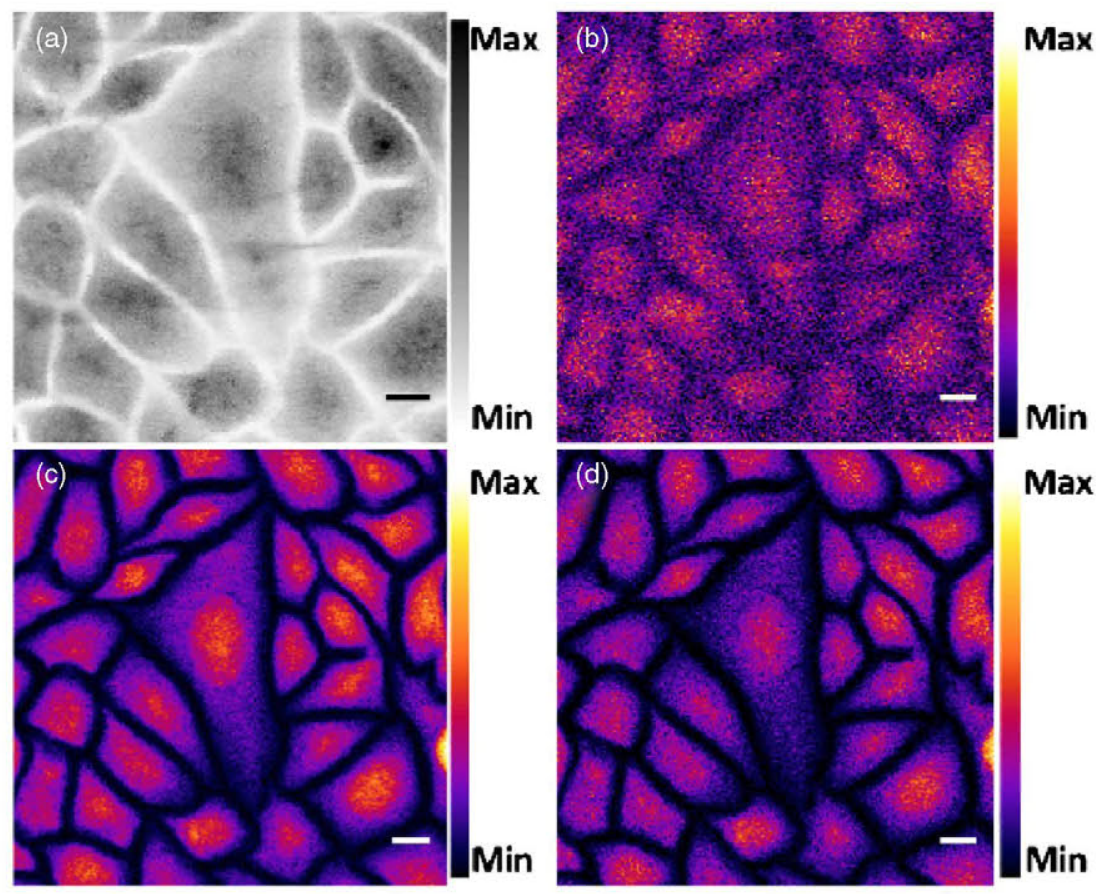

Figure 2. Ion beam analysis (IBA) on HaCaT cells: (a) STIM map showing the density of the sample, (b) RBS map showing the distribution of organic elements (C, N and O), (c) phosphorus map obtained from PIXE analysis and (d) potassium map obtained from the PIXE analysis. The RBS map shows the number of backscattered particles per pixel and PIXE maps show the $\mathrm{x}$-ray yield per pixel for the considered element. Scale bar: $10 \mu \mathrm{m}$.

Table 1. Mass fraction percentage of the main chemical elements present in a hydrated $\mathrm{HaCaT}$ cell nucleus. Elements presenting concentrations below $0.1 \%$ were at the limit of detection of the analysis techniques.

\begin{tabular}{lc}
\hline Element & Mass fraction $(\%)$ \\
\hline Hydrogen & 11.2 \\
Carbon & 19.2 \\
Nitrogen & 0.7 \\
Oxygen & 67.3 \\
Phosphorus & 0.6 \\
Potassium & 0.6 \\
Sodium & $<0.1$ \\
Magnesium & $<0.1$ \\
Silicon & $<0.1$ \\
Sulfur & $<0.1$ \\
Chlorine & $<0.1$ \\
Calcium & $<0.1$ \\
\hline
\end{tabular}

nuclei contain $70 \%$ of water and $30 \%$ of dry matter. The chemical composition used in the simulations is reported in table 1 . Since the density of the nucleus in living cells is not easily measurable and can vary in different cell lines, a density of $1 \mathrm{~g} \mathrm{~cm}^{-3}$ was considered as a reference value. Nevertheless, in order to investigate the influence of this parameter on the energy and dose deposition, several simulations were performed with densities ranging from 1 to $1.5 \mathrm{~g} \mathrm{~cm}^{-3}$. 


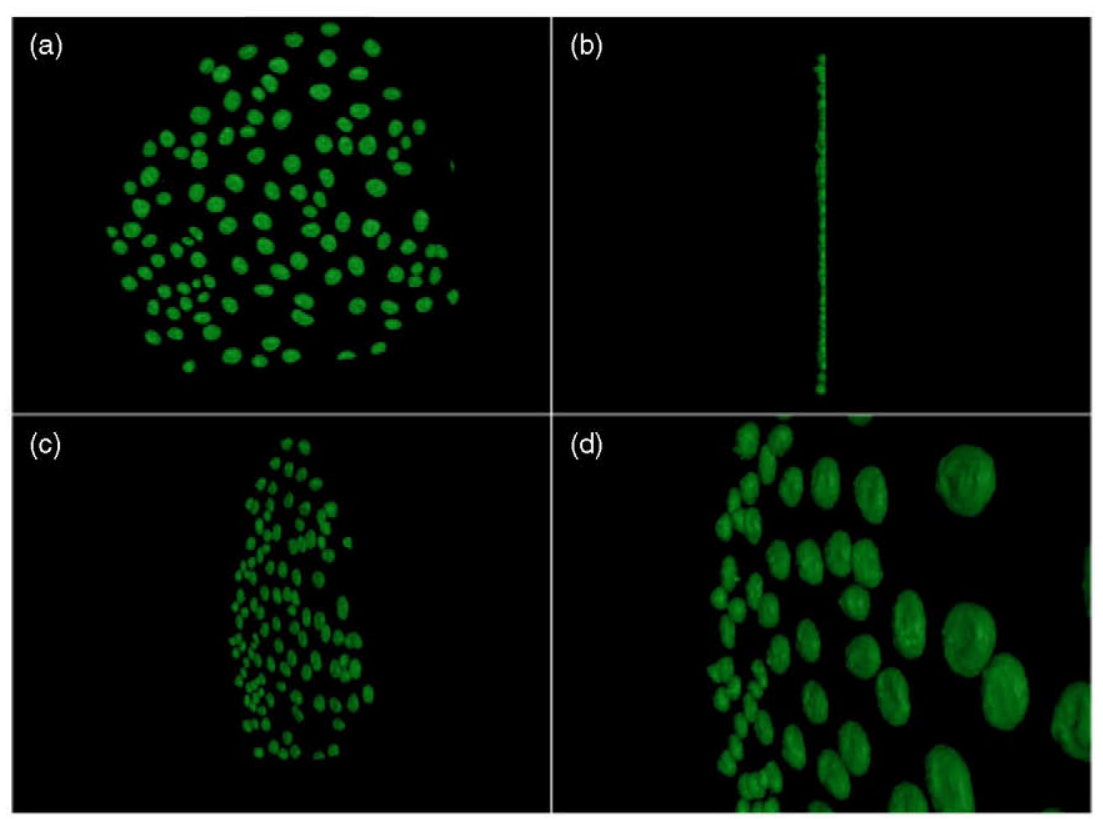

Figure 3. Isosurface view of the cell phantom used in the Geant 4 simulations. (a) Front view. The cell nuclei appear in green on a black background. Image size: $374 \times 374 \times 18 \mu \mathrm{m}^{3}$. (b) Side view of the same cell monolayer. The incoming particles are shot from the right side. (c) Tilted view. The nuclei volumes can be clearly seen. (d) Zoom on the tilted view. Images are produced using the ImageJ 3D Viewer Plugin.

\subsection{Cellular phantom reconstruction}

2.2.1. Data conversion. Images acquired from the confocal microscope were transferred into the public domain ImageJ software (http://rsbweb.nih.gov/ij/) for 3D reconstruction. An intensity threshold was then applied to separate fluorescent objects (nuclei) from the background in each slice of the stack. The cell nuclei could be extracted into an individual file containing the total number of voxels for all the nuclei in the image, the voxel size along the three dimensions, a position shift in order to centre the monolayer in the simulated irradiation dish and the list of each voxel's position and material composition, as well as the intensity fluorescence content of the voxel. We chose to name these geometries as 'cellular phantoms' in reference to human phantoms used in the medical physics community. The cellular phantom could then be imported directly into the Geant4-based simulation using the method described by Incerti et al (2009). Figure 3 shows an isosurface view of the phantom used in this work.

2.2.2. Identification of each nucleus. The method previously reported in Incerti et al (2009) was used. Briefly, the cell nuclei have been modelled in the simulation using a phantom made of parallelepiped voxels. Each individual voxel contains information about its absolute position within the whole cellular phantom as well as information about local chemical composition and density. The 'pure' parameterized approach based on specific geometry classes available in the Geant4 toolkit was used. In this classical approach, the cell phantom is made of multiple copies of a parallelepiped voxel, all having the same geometry. Additional functionalities were added to the previously reported method as a multi-cellular phantom 
requires considering each nucleus present in the image as an independent object. Indeed, this step is mandatory to calculate the energy and specific energy deposition in each nucleus individually. For this purpose, automatic object recognition was performed on the $z$-stacks using the '3D object counter' plug-in developed by Bolte et al for ImageJ (Bolte et al 2006). This plug-in provides geometrical information on each cell nucleus in the image, such as the position of the barycentre and the volume. The calculation of the total energy deposited in every nucleus from the energy deposited in single voxels was made possible by a modification of the $3 \mathrm{D}$ object counter plug-in in order to produce a file containing the position and the number of the parent cell nucleus for every voxel constituting the image. After processing the images with the modified 3D object counter plug-in, the data obtained were stored in the ASCII format and used in the Geant 4 simulations to calculate the specific energy deposited in every single nucleus. Mis-recognized cells (i.e. double nuclei counted as one or cells cut by the image border) were removed from the list of recognized cells.

\subsection{Geant4 physics processes}

The most recent public release of Geant 4 was used for this work (release 9.4). In the MeV range, the dominant physical processes affecting alpha particles are multiple scattering and ionization. Multiple scattering was simulated using the G4hMultipleScattering class and ion ionization was simulated using the G4ionIonisation class. Secondary ionization electrons were tracked using the so-called Livermore set of models for ionization and Bremsstrahlung, described by the G4LivermoreIonisationModel and G4LivermoreBremsstrahlungModel classes respectively, and taking into account the multiple scattering process, simulated by the G4eMultipleScattering class. Further details on these classes might be found in the Geant 4 documentation (http://geant4.org). The default cut-off for the production of secondary particles has been set to $1 \mu \mathrm{m}$ and a maximum step limiter has been used as previously explained (Incerti et al 2009).

\subsection{Description of the irradiation facilities modelled in Geant4}

Two irradiation facilities have been developed for cell irradiation: a microbeam used for dose controlled targeted irradiation and a ${ }^{239} \mathrm{Pu}$ alpha particle source used for random irradiation mainly for protocol testing experiments.

2.4.1. Microbeam. The microbeam irradiation facility has been described in detail previously (Barberet et al 2005). This set-up allows us to achieve a lateral resolution of $1 \mu \mathrm{m}$ under vacuum for alpha particles and proton beams with energies up to $3 \mathrm{MeV}$. After extraction in air through a low pressure gas detector, a beam spot size of $10 \mu \mathrm{m}$ diameter is achieved and a controlled number of particles are delivered on single cells. This beam line has been fully modelled in the Geant4 advanced example mentioned previously. In this work, only the key parts, i.e. the parts having a significant influence on the beam energy and size at the cell monolayer position, were carefully modelled in Geant4. The primary microbeam (at the focal position of the beam optics, in vacuum) was modelled as mono-energetic $3 \mathrm{MeV}$ alpha particles distributed on a Gaussian distribution presenting a $1 \mu \mathrm{m}$ full width at half maximum (FWHM). As the beam extraction stage (gas detector, residual air layer and cell culture support) has an influence on the beam energy and size at the position of the cell monolayer, all the materials traversed by the particles have been taken into account. Prior to hitting the cell nuclei, the beam traverses the following material layers: $4 \mathrm{~mm}$ of butane at 10 mbar, a $150 \mathrm{~nm}$ thick $\mathrm{Si}_{3} \mathrm{~N}_{4}$ window, a residual air layer estimated to be $200 \mu \mathrm{m}$ thick and a $3.8 \mu \mathrm{m}$ thick polypropylene foil used 

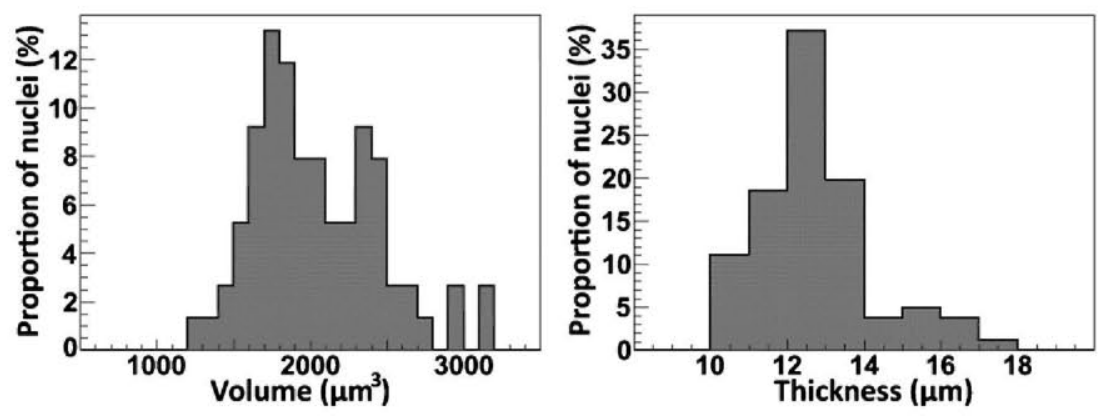

Figure 4. Distribution of nuclear volumes and thicknesses extracted from the cellular phantom using the 3D object counter plug-in for ImageJ.

as a cell culture support. For radiobiology experiments, the cell dish was designed to keep the cells fully immersed in their living medium. In the case of Geant4 simulations, the cellular phantom was embedded in liquid water. The layout of the micro-irradiation set-up is shown in figure 1 .

2.4.2. ${ }^{239} \mathrm{Pu}$ alpha particle irradiator. The set-up described in figure 1 was fully simulated in Geant4. Compared to the microbeam irradiation simulations, only the particle generator was modified to generate particle trajectories randomly. The alpha particles are shot in $4 \pi$ steradians from a flat circle of $1.5 \mathrm{~cm}$ diameter. The emitting surface is situated $1 \mathrm{~mm}$ away from the cell monolayer. The design of the cell dish, i.e. the cell support, the cellular phantom and the medium is similar to the one used for the simulations of microbeam irradiations. The total number of particles shot in $4 \pi$ steradians was calculated in order to correspond to irradiation times of 5,10 and $30 \mathrm{~min}$.

\section{Results}

\subsection{Cellular phantom properties}

The combination of confocal microscopy and IBA at the sub-micrometre scale provides quantitative data concerning the volumes, thicknesses and chemical composition of the HaCaT cell nuclei. Image processing on the confocal image shown in figure 3 gives the distribution of volumes and thicknesses of the nuclei present in the image as shown in figure 4 . The volume distribution shows that, in a typical $\mathrm{HaCaT}$ cell population, the nuclear volumes range from about 1000 to $3000 \mu \mathrm{m}^{3}$ with a mean value of $2047 \mu \mathrm{m}^{3}$. The thickness of the cell nuclei as provided by the $3 \mathrm{D}$ object counter plug-in represents the depth of the bounding box defined around each nucleus. It thus represents a maximum thickness since the nuclei do not show a homogeneous thickness as can be seen in figures 3(b) and (d). The distribution of thicknesses ranges from 10 to $18 \mu \mathrm{m}, 85 \%$ of the nuclei having a thickness between 10 and $14 \mu \mathrm{m}$. The mean thickness obtained from the distribution is $12.8 \mu \mathrm{m}$.

IBA performed on a monolayer of $\mathrm{HaCaT}$ cells provides the mean areal mass of the cell nuclei (using STIM) as well as the proportion of major and trace elements (RBS and PIXE). Figure 2 illustrates typical maps obtained using these techniques. Compared to previously reported maps (Incerti et al 2009, Simon et al 2011), the lateral resolution of the ion beam has been improved. The identification of the main sub-cellular compartments is thus easier. 
In addition, the areal mass measurement of the cell nuclei was carried out using the STIM technique which provides more accurate results as reported by Devès and Ortega (2002). A mean areal mass of $119 \mu \mathrm{g} \mathrm{cm}^{-2}$ (dry mass) has been obtained from 16 cell nuclei selected on the maps shown in figure 2 . The absolute mass fraction obtained for the major elements are summarized in table 1 . These measurements have been carried out on two different experiments on about 200 cells.

Mass fractions in hydrated samples were extrapolated from measurements on dry samples by considering that a wet cell contains $30 \%$ of dry matter and $70 \%$ of water. The values reported in table 1 show that a hydrated $\mathrm{HaCaT}$ nucleus mainly contains oxygen, carbon and hydrogen. In the case of low concentration elements, only nitrogen, phosphorus and potassium were taken into account in the simulations since they present concentrations significantly above the limit of detection. Other trace elements (sodium, magnesium, silicon, sulfur, chlorine and calcium) were detected using PIXE but were neglected in the simulations since they present concentrations below $0.1 \%$.

The data presented in table 1 differ significantly from the chemical composition reported in Incerti et al (2009). This difference comes from the choice of a different carbon/hydrogen ratio. Indeed, the IBA techniques used in this work do not provide the amount of hydrogen present in the sample, as this element is too light to be measured using backscattering. Therefore, a fixed carbon/hydrogen ratio is usually assumed based on published values. The carbon/hydrogen ratio of $\mathrm{C}_{1.25 x} \mathrm{H}_{x}$ assumed in Incerti et al (2009) was based on previously reported data, obtained in the case of cancer cells (Michelet and Moretto 1999) and available at that time. In this work, a carbon/hydrogen ratio of $\mathrm{C}_{x} \mathrm{H}_{2 x}$ was used. This ratio was chosen to follow the ICRP recommendations (Snyder et al 1975), and confirmed in recently published work (Carmona et al 2008). Except for that difference, major and trace elements were found to be in relatively similar amount, the difference being below $10 \%$ between the two measurements for oxygen, phosphorus and potassium when comparing data presented here and data from 2009. The amount of nitrogen is also found to be lower in this work. This difference can be explained by a slightly higher amount of oxygen, having a strong impact on other concentrations since it represents more than $60 \%$ of the mass of a hydrated cell nucleus. The slight increase in oxygen concentration could come from variability in the cryofixation/lyophilization process or from a higher precision in defining the nucleus on the elemental maps.

Significant discrepancies have been found in cellular compositions recommended by the ICRU report 44 (ICRU 1989), the ICRP (Snyder et al 1975), the data published by Alard et al (2002) and the data reported in Incerti et al (2009). The influence of these discrepancies on the calculation of energy deposition and specific energy per cell is nevertheless relatively small (Incerti et al 2009). The parameter having the stronger influence to this respect is the density of the tissue as it impacts the number of interactions and the mass of the target volume. The influence of the cellular density on the results is further discussed in section 3.4.

\subsection{Quantification of energy and specific energy deposition}

The dosimetry method used in this work provides the number of hits, the total absorbed energy (in $\mathrm{MeV}$ ) and specific energy (in Gy) for every nucleus present in the confocal image. Distributions of these quantities, in the case of typical irradiations using the microbeam and the ${ }^{239} \mathrm{Pu}$ irradiator are shown in figures 5 and 6 , respectively. These distributions provide the mean value and the variation of these quantities, presented hereafter in terms of root mean square.

In the case of targeted microbeam irradiation, simulations have been performed with 1 , 5 and 50 incoming $3 \mathrm{MeV}$ alpha particles. The actual mean number of hits measured in the 

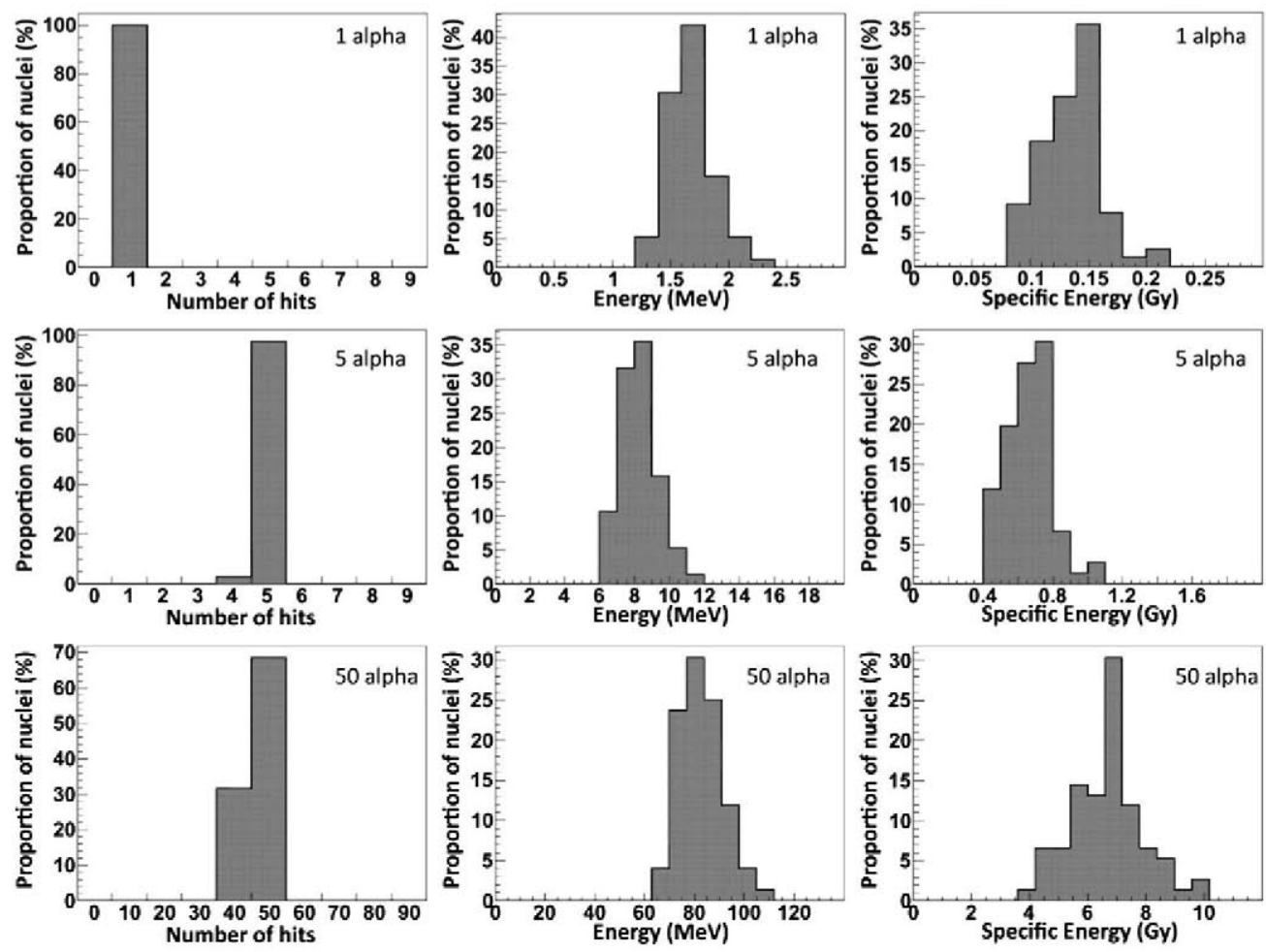

Figure 5. Number of hits, total energy deposition and specific energy per nucleus in the case of targeted irradiation. First line: 1 alpha particle delivered per nucleus, second line: 5 alpha particles delivered per nucleus, third line: 50 alpha particles delivered per nucleus.

cellular phantom was $1,4.97 \pm 0.16$ and $49.58 \pm 0.75$, respectively. The two latter values indicate that about $0.6 \%$ of the particles are scattered at large angles and therefore miss the targeted nuclei. The mean energy delivered to the nuclei in the case of 1 alpha particle is $1.671 \pm 0.189 \mathrm{MeV}$ which leads to a mean specific energy of $0.135 \pm 0.025 \mathrm{~Gy}$. In the cases of 5 and 50 incoming particles, the mean absorbed energies were found to be $8.284 \pm 0.988$ and $82.810 \pm 8.729 \mathrm{MeV}$, respectively. These values are very close to the ones that can be calculated by multiplying the number of incoming particles with the mean energy deposited by 1 particle, indicating a weak variation of the energy deposited from 1 particle to the other. The influence of the few particles scattered off axis can also be neglected.

Random irradiations using a ${ }^{239} \mathrm{Pu}$ source show much wider energy and specific energy distributions. The simulations show that irradiation of about $5 \mathrm{~min}$ is sufficient to achieve a mean of more than one traversal per nucleus $(1.421 \pm 1.150)$. At this level of exposure, a Poisson-like distribution is obtained for the energy deposition and specific energy. Increasing the exposure time to 10 and 30 min leads to the mean number of hits per nucleus of $2.697 \pm 1.496$ and $7.658 \pm 3.378$, respectively. When the irradiation time is increased to more than $30 \mathrm{~min}$, these distributions tend to become Gaussian. This statistical effect is similar to the one observed when simple cell geometries are used. Nevertheless, the use of realistic cell geometries provides more realistic values for the mean absorbed energy and specific energy in every cell nucleus. 

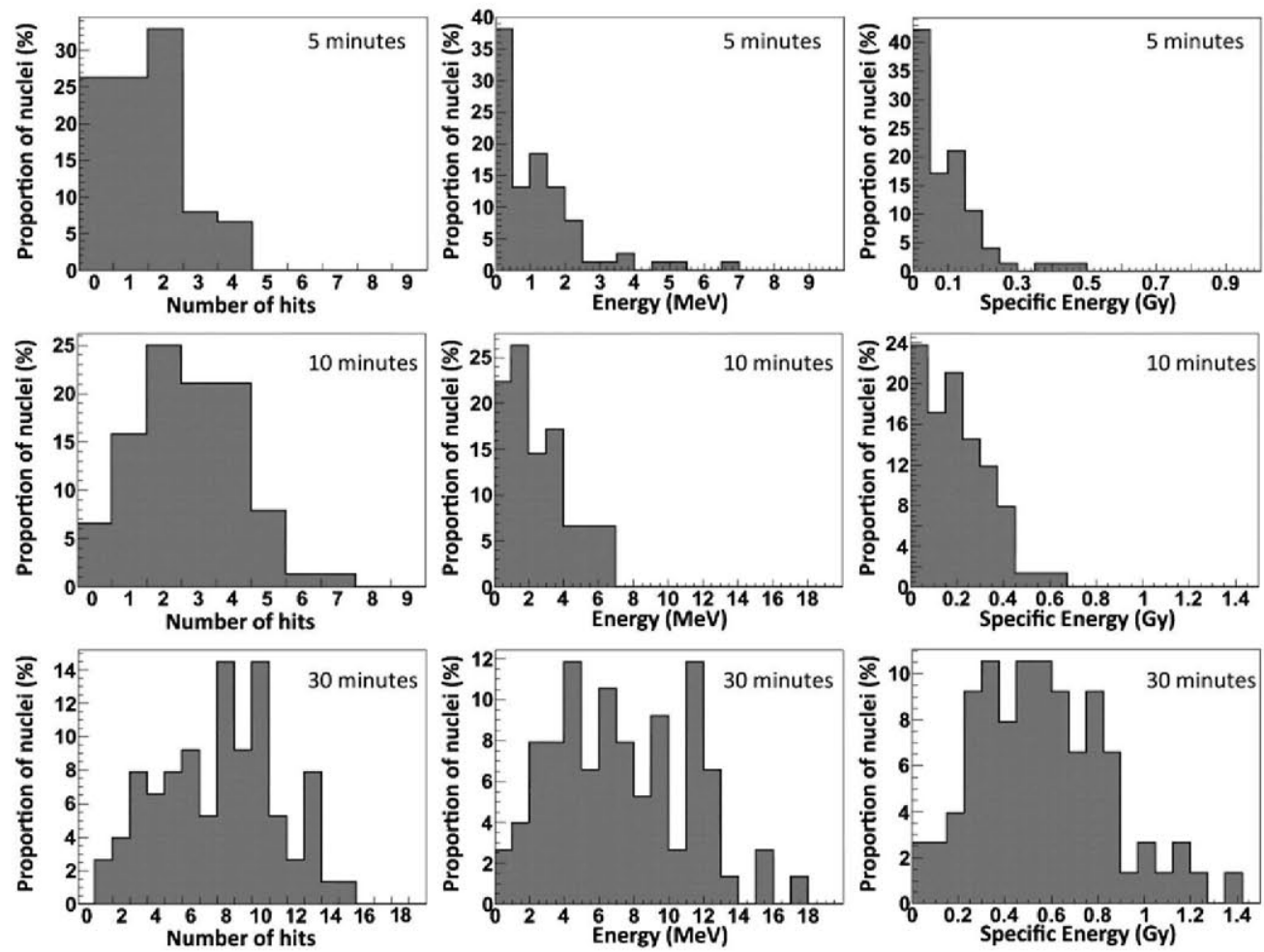

Figure 6. Number of hits, total energy deposition and specific energy per nucleus in the case of random irradiation. First line: $5 \mathrm{~min}$ exposure, second line: $10 \mathrm{~min}$ exposure, third line: $30 \mathrm{~min}$ exposure.

\subsection{Qualitative images of energy deposition}

The ability of Geant 4 to track particles in individual voxels allows the reconstruction, in three dimensions, of the energy deposition along the ion tracks. Arbitrary colours were attributed to the energy deposited in the voxels constituting the cellular phantom and qualitative images of the ion tracks in the nuclear volumes were produced. Typical images are shown in figure 7 in the case of targeted irradiation with 50 alpha particles (figure 7(a)) and $5 \mathrm{~min}$ random irradiation (figure 7(b)). In the case of targeted irradiation, the energy is deposited in tracks perpendicular to the cell monolayer support. The 50 hits are concentrated in the central area of the nuclei and about $99 \%$ of the particles are delivered in a circle of $10 \mu \mathrm{m}$ diameter, corresponding to the beam size at the position of the cell monolayer. The random irradiation leads to a more complex energy deposition pattern. Since the radioactive source has a diameter much bigger than the cell monolayer, the nuclei can also be hit by alpha particles at grazing angles. This leads to ion tracks presenting a small angle with the cell support as can be seen in figure $7(b)$.

The main interest of these qualitative images of energy deposition along the ion tracks is that they can be compared to experimental data. Indeed, immuno-labelling techniques provide an indirect visualization of the energy deposition pattern by means of DNA double strand break signalling and repair proteins (Aten et al 2004, Hauptner et al 2006, Jakob et al 2003). Such images are shown in figure 8 for typical microbeam and source irradiations. 


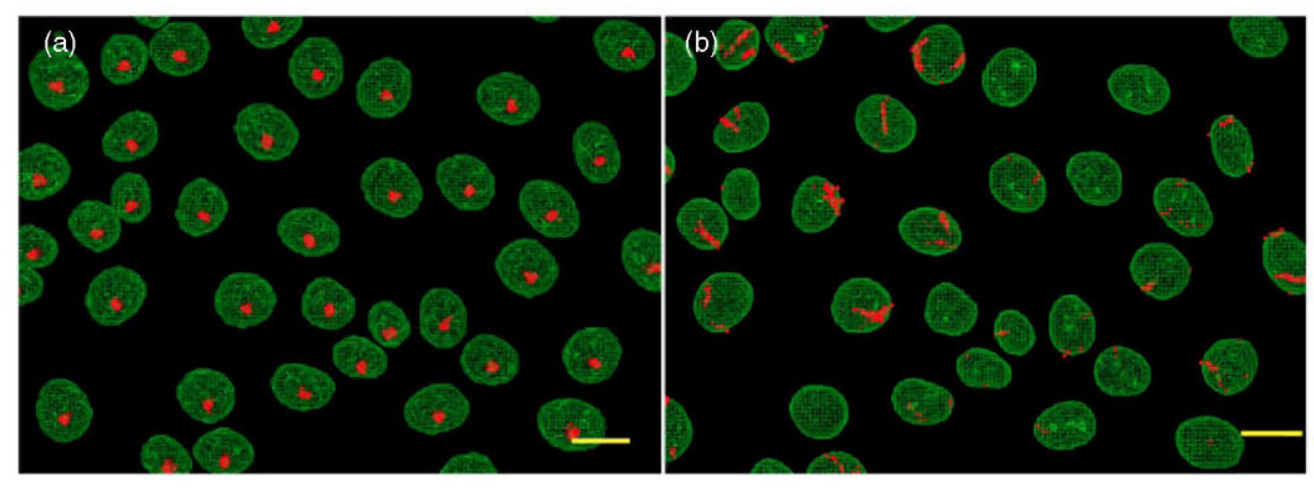

Figure 7. Isosurface view of the cellular phantom after irradiation. (a) Targeted irradiation with 50 alpha particles. (b) 10 min random irradiation using the ${ }^{239} \mathrm{Pu}$ source. The cell nuclei appear in transparent green. Energy deposition appears in red. Intensity normalization has been performed on the red channel. Scale bar: $20 \mu \mathrm{m}$.

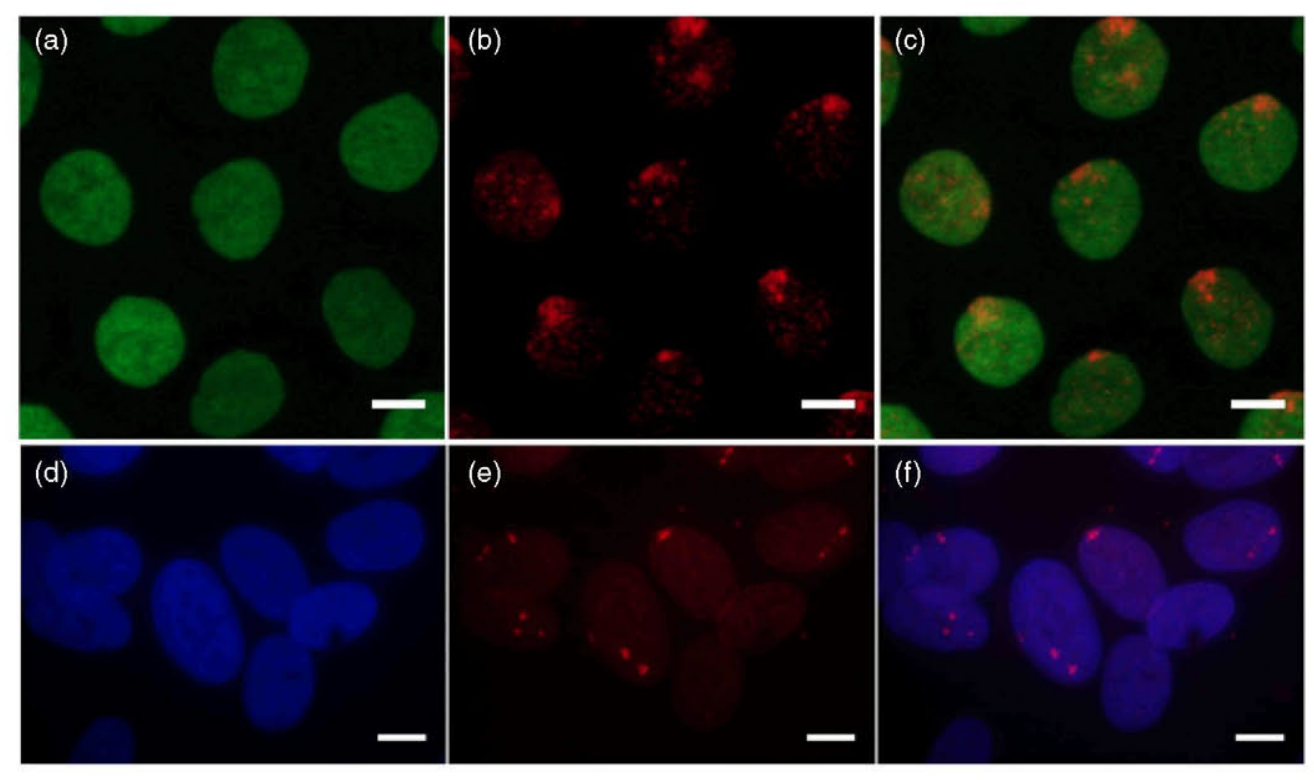

Figure 8. Fluorescent images of HaCaT keratinocyte nuclei showing DNA double strand break signalling proteins after typical exposures to alpha particles. The first row shows cells targeted with 50 alpha particles, and the second row shows cells exposed 5 min to the ${ }^{239} \mathrm{Pu}$ source. (a) H2B-GFP delimiting the nuclear areas, (b) H2A.X-Pser139 indicating the presence of radiation-induced DNA double strand breaks, (c) Merge, (d) Hoechst ${ }^{33342}$ delimiting the nuclear areas, (e) ATM-Pser1981, (f) Merge. Scale bar: $10 \mu \mathrm{m}$.

It has to be noted that the cells used in the Monte-Carlo simulations and the cells irradiated experimentally are from different samples that were prepared under similar conditions.

In the case of random irradiation, damaged sites showing different shapes can be observed and seem correlated with the energy deposition patterns observed in figure 7(b). Small spots such as ATM-P ${ }^{\text {ser1981 }}$ foci as well as aligned foci suggesting energy deposition along a track can be observed. 

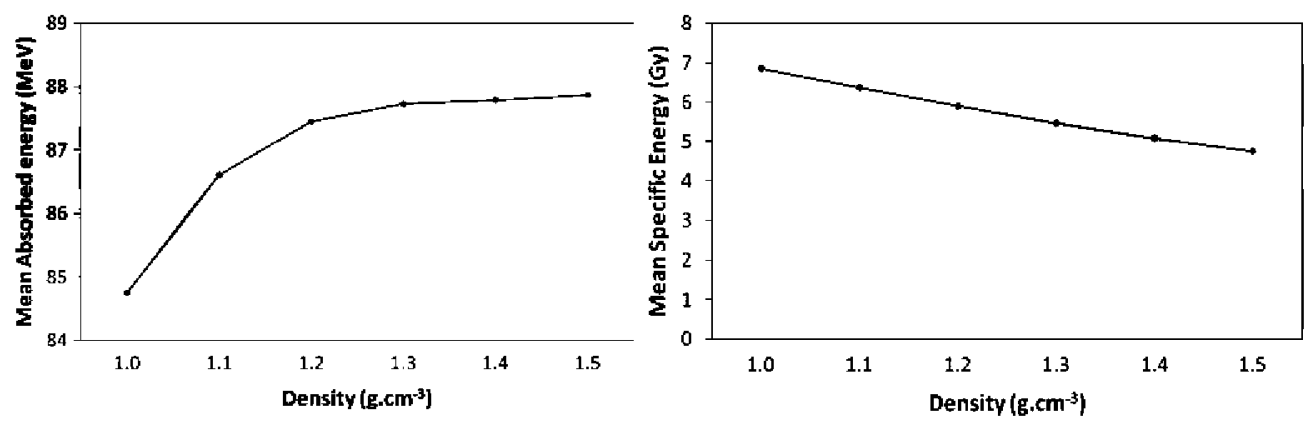

Figure 9. Evolution of the mean energy deposition and mean specific energy as a function of the nuclei density. The cell nuclei were targeted and irradiated with 50 alpha particles.

\subsection{Influence of the nucleus density}

Since the density of living cell nuclei is not precisely known, different values were used to estimate the influence of this parameter on the energy and specific energy deposition. For a dosimetry purpose, living cells are often considered as water-filled volumes. The density used in most of the studies is thus usually $1 \mathrm{~g} \mathrm{~cm}^{-3}$ (water density). In this work, results obtained with densities ranging from 1 to $1.5 \mathrm{~g} \mathrm{~cm}^{-3}$ were compared to investigate the influence of this parameter on the absorbed energy and specific energy. Figure 9 summarizes the mean absorbed energy and specific energy in the case of targeted irradiation with 50 alpha particles for the different values of nuclei density.

As expected, a higher nuclear density led to a higher energy deposition in the ion tracks and thus to a higher total energy deposition in the cell nuclei. This result can easily be understood since the number of physical interactions between the incident particles and the atoms constituting the nuclei is higher. Interestingly, the specific energy per nucleus does not increase at higher nuclei densities and even decreases slightly as the density increases. This effect can be explained by an increase in the cellular masses (used to calculate the specific energy in Grays from the energy deposition) that overcomes the increase in the energy deposition.

\section{Discussion}

\subsection{Absorbed energy and specific energy calculations}

The energy and specific energy distributions shown in figures 5 and 6 provide information on the variation of these quantities from one cell nucleus to the other. The aim of targeted irradiation using a microbeam is usually to deliver a controlled number of ions in a well-defined cell compartment. This is achieved by the use of particle detectors counting the number of ions delivered on target. The energy distribution shown in figure 5 indicates that, even if the number of ions delivered on each cell nucleus is perfectly controlled, the total absorbed energy and specific energy can vary from 1.2 to $2.5 \mathrm{MeV}$ per incoming particle. This result underlines that detailed modelling of the cell morphology is critical to obtain realistic values of the energy deposition when low energy incoming ions are considered. In order to check that the mean values of the specific energy obtained in this work are consistent with previously reported values, they were compared to the values reported in Incerti et al (2009) in the case of irradiations with similar incoming energies and geometry. This comparison is reported in table 2 . 
Table 2. Mean specific energy absorbed in cell nuclei for one hit. The first column summarizes the values calculated in this work for $3 \mathrm{MeV}$ incoming alpha particles. The second column reports the value reported in Incerti et al (2009) for a cell nucleus with a similar shape and size.

\begin{tabular}{lll}
\hline & This work & Incerti et al (2009) \\
\hline Geometry & Voxelized & Voxelized \\
Mean nucleus volume $\left(\mu \mathrm{m}^{3}\right)$ & 2047 & 1373 \\
Incoming energy $(\mathrm{MeV})$ & 3 & 3 \\
Mean specific energy $(\mathrm{Gy})$ & 0.13 & 0.08 \\
\hline
\end{tabular}

Even if a direct comparison is made difficult by differences in the modelling (i.e. single cell versus multiple cells and chemical composition), the data presented in table 2 show reasonable agreement. The mean specific energy obtained on 76 cells is higher than the one obtained on a single cell. Nevertheless, as reported in figures 5 and 6 , the specific energy absorbed from one nucleus to the other in a population can vary by a factor of more than 2 due to variations in its geometry, indicating that the single cell modelled in Incerti et al (2009) is fitting well in the distribution obtained for a cell population.

The irradiations using a radioactive source presented in this work show much wider energy and specific energy distributions as can be expected from the randomness of the irradiation process. In that case, the path of the ions through the cell nuclei is not necessarily perpendicular to the cell monolayer and the path length can vary a lot from one incoming particle to the other. This can be seen in figure 7(b) where the incoming angle is suggested by the length of the observed track. Detailed modelling of the cell nuclei geometry is thus critical to obtain realistic values on the total amount of energy deposited in each nucleus.

The values obtained in this work for specific energy per cell are difficult to compare directly with the one found in the literature (Roeske et al 2007, Elbast et al 2011) because of different alpha particle energies, cell geometries and irradiation geometries. Nevertheless, good agreement in the orders of magnitude can be found indicating that a few MeV incoming alpha particle traversing a cell nucleus corresponds to a specific energy between 0 and $0.25 \mathrm{~Gy}$. This value strongly depends on the nucleus volume and on the path of the particle through the nucleus underlining the importance of modelling realistic geometries.

The main limitation of the method applied in this work is the modelling of the cell nuclei as volumes with a homogeneous composition. Taking into account the complex geometry and composition of the chromatin inside the nuclei would be of great interest to improve the simulations.

\subsection{Correlating simulated energy deposition and damage observed experimentally}

The mapping of energy deposition in the three dimensions provided by the simulations gives the possibility to address the challenge of correlating the energy deposition to the radio-induced DNA damage. Using the method described in this work, it is possible to obtain images showing the energy deposition along the ion tracks. In parallel, images of DNA damage signalling proteins have been obtained experimentally using immunocytofluorescence.

These two types of images, even if they are obtained on different samples, show rather similar patterns, indicating a correlation between the energy deposition and the biological effect. The biological damage (DNA double strand breaks) patterns detected look rather similar to the energy deposition patterns obtained using Geant4. For microbeam irradiations, the damaged areas are located on the nuclei periphery and show a bigger diameter compared to the area where the energy is deposited according to the simulation. These effects can be 
explained by the targeting accuracy of the experimental facility, leading to a few micrometres' shift between the targeted point and the actually irradiated position, and the beam scattering that may be more important as compared to the simulations because of a slightly higher pressure in the gas detector or a thicker residual air layer. The influence of the biological processes such as chromatin mobility (Jakob et al 2003) or the molecular size of the H2A.X-P ${ }^{\text {ser139 }}$ domains can also explain this difference. Indeed, the difference between energy deposition at the nanometre scale, the track structure (clustered ionization) and the DNA damage distribution or definition (H2A.X-Pser139 foci) has to be taken into account. It is actually assumed in the literature that one DNA double strand break leads to the phosphorylation of around 2000 histones H2A.X-Pser139 (Olive et al 2004, Pilch et al 2003, Rogakou et al 1998).

When the longer tracks are considered, differences can be observed (i.e. for random irradiations). Indeed, while the simulations show a relatively continuous energy deposition along the tracks, experimental observations of DNA double strand breaks are more discontinuous. These discrepancies can be explained by a heterogeneous distribution of DNA inside the cell nuclei (Jakob et al 2003). Moreover, clustering of H2A.X-Pser139 chromosome domains has been reported after alpha particle irradiation (Aten et al 2004). A better description of the experimental parameters in the simulations will require taking into account more precisely the complex geometry, heterogeneity and composition of the chromatin in the simulations.

\subsection{Influence of the nuclei density and specific energy at the cellular scale}

The density of the cell nuclei used in this work is not precisely known and is most probably not homogeneous as can be seen in the STIM map presented in figure 2(a). For this reason, several values of the nuclei density were used to investigate the effect of this parameter on the energy deposition in the nuclei. The obtained results show that it is possible to deposit more energy in a given volume and thus create more molecular damage while the specific energy decreases. This effect is due to the mass normalization in the calculation of the specific energy, the total mass of a nucleus increasing faster with the density than the energy deposited by an incoming particle. This can thus lead to difficulties in establishing a dose-effect relationship when irradiations are performed at the cellular scale. It underlines that the concept of specific energy and absorbed dose has to be used very carefully for characterizing experiments at the cell level.

\subsection{Limitations of the method}

The dosimetry method presented in this work was developed to take into account realistic cellular characteristics in Monte-Carlo simulations. Nevertheless, the approach proposed here encounters some limitations that will have to be addressed to go further in the design of robust microdosimetric methods on fully realistic objects.

- The volumes of the cell nuclei are relatively easy to obtain from confocal microscopy since they can be labelled with fluorescent markers and identified using 3D image processing. The segmentation of cytoplasms is much more difficult since cytoplasms from different cells are touching each other are cannot be separated easily. The present method was tested only on cell nuclei up to now and extending it to a complete cellular volume will require the development of specific image processing techniques.

- The use of a realistic cellular composition is limited by the discrepancies of the standard cellular compositions found in the literature. The determination of the concentrations of major and trace elements in the cellular compartments is possible using IBA techniques 
but the amount of hydrogen is difficult to measure using these techniques and is usually extrapolated using tabulated values. Further work is thus needed to obtain more precise data about the cellular composition.

- The cell nuclei used in the simulations are filled with homogeneous materials and a more precise description of the chromatin heterogeneity is necessary to correlate the energy deposition pattern and the DNA damage observed experimentally.

\section{Conclusions}

The work presented here provides a complete dosimetric characterization of two alpha particle irradiation devices: a microbeam and a ${ }^{239} \mathrm{Pu}$ radioactive source. For this purpose, the methodology previously reported in Incerti et al (2009) has been extended to allow the energy and specific energy calculation for every cell nucleus present in a multi-cellular phantom. Moreover, the energy deposition patterns inside the nuclei are extracted as qualitative images and compared with DNA damage observed using immuno-staining experiments. These patterns show a correlation between the energy deposition patterns and the radiation-induced $\gamma-\mathrm{H} 2 \mathrm{~A} . \mathrm{X}$ foci observed in vitro, opening thus interesting features for dose-effect relationship studies. The mean specific energy per cell nucleus obtained from the simulations on a cell monolayer is in good agreement with the value obtained for one cell reported in Incerti et al (2009) and with other published data even if a direct comparison is hindered by the differences in the cell geometry modelling and in the incoming particle energies. The cellular chemical composition measured in this work is mainly similar to previously reported ones for most of the elements. Nevertheless, discrepancies in the literature about the hydrogen content (that could not be measured here) indicate that further work is needed to obtain more precise data about the complete cellular composition.

The use of open-source software for 3D imaging and segmentation as well as for MonteCarlo simulations makes the methodology presented here an ideal tool to simulate different irradiation experiments. It can for example be used to simulate different types of ionizing radiations ( $\mathrm{x}$ - or gamma rays, beta rays or heavier ions) according to the capabilities of the Geant 4 simulation toolkit to track these particles. In addition, other cell lines, presenting different geometries, could also be modelled using the method presented here. For this purpose, cell lines expressing markers adapted to confocal microscopy that can be used to define the cellular volume have to be developed.

Finally, the authors think that the approach presented in this paper could help to address issues related to small scale dosimetry in the frame of targeted radiation immunotherapy. Indeed, for particle emitters used in radiation therapy, the dose delivered to the tissues has to be determined at a scale comparable to the cell size, leading thus to a strong influence of the cellular volume.

\section{Acknowledgments}

The authors would like to acknowledge P Legros and C Poujol from the Bordeaux Imaging Center for their helpful and friendly assistance in fluorescence microscopy techniques. Special thanks to L Daudin and Ph Alfaurt for their technical support and assistance. The AIFIRA facility and the technical development of the microbeam line have been financially supported by the Région Aquitaine and partially supported by the European Community as an Integrating Activity 'Support of Public and Industrial Research Using Ion Beam Technology (SPIRIT)' under EC contract no 227012. These activities are partly funded by the French Agence 
Nationale de la Recherche Contract No. ANR-09-BLAN-0135-01. FV is partially supported by IRSN (Institut de Radioprotection et de Sûreté Nucléaire).

\section{References}

Agostinelli S et al 2003 Geant4-a simulation toolkit Nucl. Instrum. Methods A 506 250-303

Alard J A et al 2002 Simulation of neutron interactions at the single-cell level Radiat. Res. 158 650-6

Apostolakis J et al 2009 Geometry and physics of the Geant 4 toolkit for high and medium energy applications Radiat. Phys. Chem. 78 859-73

Aten J A, Stap J, Krawczyk P M, van Oven C H, Hoebe R A, Essers J and Kanaar R 2004 Dynamics of DNA double-strand breaks revealed by clustering of damaged chromosome domains Science 303 92-5

Barberet P, Balana A, Incerti S, Michelet-Habchi C, Moretto P and Pouthier T 2005 Development of a focused charged particle microbeam for the irradiation of individual cells Rev. Sci. Instrum. 76 1-6

Barberet P, Daudin L, Gordillo N, Sorieul S, Simon M, Seznec H, Idarraga I, Incerti S, Balana A and Moretto P 2011 First results obtained using the CENBG nanobeam line: performances and applications Nucl. Instrum. Methods B $2692163-67$

Beaton L A, Burn T A, Stocki T J, Chauhan V and Wilkins R C 2011 Development and characterization of an in vitro alpha radiation exposure system Phys. Med. Biol. $563645-58$

Bolte S and Cordelières F P 2006 A guided tour into subcellular colocalization analysis in light microscopy J. Microsc. $224213-32$

Boukamp P, Petrussevska R, Breitkreutz D, Hornung J, Markham A and Fusenig N 1988 Normal keratinization in spontaneously immortalized aneuploid human keratinocyte cell line J. Cell Biol. 106 761-71

Carmona A, Devès G and Ortega R 2008 Quantitative micro-analysis of metal ions in subcellular compartments of cultured dopaminergic cells by combination of three ion beam techniques Anal. Bioanal. Chem. 390 1585-94

Champion C, Zanotti-Fragonara P and Hindié E 2008 CELLDOSE: a Monte Carlo code to assess electron dose distribution-S values for ${ }^{131} \mathrm{I}$ in spheres of various sizes J. Nucl. Med. 49 151-7

Dadachova E 2010 Cancer therapy with alpha-emitters labelled peptides Semin. Nucl. Med. 40 204-8

Devès G and Ortega R 2002 Subcellular mass determination by ${ }^{4} \mathrm{He}^{+}$energy-loss micro-spectrometry Anal. Bioanal. Chem. $374390-4$

Elbast M, Saudo A, Franck D, Petitot F and Desbrée A 2011 Microdosimetry of alpha particles for simple and 3D voxelised geometries using MCNPX and Geant4 Monte Carlo codes Radiat. Prot. Dosim. (online first: dx.doi.org/10.1093/rpd/ncr401)

Fokas E, Kraft G, An H and Engenhart-Cabillic R 2009 Ion beam radiobiology and cancer: time to update ourselves Biochem. Biophys. Acta 1796 216-29

Hauptner A et al ed P Sigmund 2006 Spatial distribution of DNA double-strand breaks from ion tracks Ion Beam Science: Solved and Unsolved Problems (Copenhagen: Royal Danish Academy of Sciences and Letters) pp 5985 (Available at http://www.e12.physik.tu-muenchen.de/groups/rim/papers/hauptner-mfm-2.pdf)

ICRU 1989 Tissue substitutes in radiation dosimetry and measurements ICRU report no 44 (Washington, DC: International Commission for Radiation Units and Measurements)

ICRU 1998 Fundamental quantities and units for ionizing radiation ICRU report no 60 (Washington, DC: International Commission for Radiation Units and Measurements)

Incerti S, Seznec H, Simon M, Barberet P, Habchi C and Moretto P 2009 Monte Carlo dosimetry for targeted irradiation of individual cells using a microbeam facility Radiat. Prot. Dosim. 133 2-11

Jakob B, Sholz M and Taucher-Sholz G 2003 Biological imaging of heavy charged-particle tracks Radiat. Res. 159 676-84

Kanda T, Sullivan K F and Wahl G M 1998 Histone-GFP fusion protein enables sensitive analysis of chromosome dynamics in living mammalian cells Curr. Biol. 8 377-85

Michelet C and Moretto P 1999 3D mapping of individual cells using a proton microbeam Nucl. Instrum. Methods B $150173-8$

Miller J H, Chrisler W B, Wang X and Sowa M B 2011 Confocal microscopy for modeling electron microbeam irradiation of skin Radiat. Environ. Biophys. 50 365-9

Mulford D A, Scheinberg D A and Jurcic J G 2005 The promise of targeted $\alpha$-particle therapy J. Nucl. Med. 46 199-204

Nikjoo H, Ueharab S, Emfietzoglou D and Cucinotta F A 2006 Track structure codes in radiation research Radiat. Meas. 41 1052-74

Nikjoo H, Uehara S, Wilson W E, Hoshi M and Goodhead D T 1998 Track structure in radiation biology: theory and applications Int. J. Radiat. Biol. 73 355-64 
Olive P L and Banath J P 2004 Phosphorylation of histone H2AX as a measure of radiosensitivity Int. J. Radiat. Oncol. Biol. Phys. 58 331-5

Pilch D R, Sedelnikova O A, Redon C, Celeste A, Nussenzweig A and Bonner W M 2003 Characteristics of $\gamma$-H2AX foci at DNA double-strand breaks sites Biochem. Cell. Biol. 81 123-9

Roeske J C, Aydogan B, Bardies M and Humm J L 2008 Small-scale dosimetry: challenges and future direction Semin. Nucl. Med. 38 367-83

Roeske J C and Hoggarth M 2007 Alpha-particle Monte Carlo simulation for microdosimetric calculations using a commercial spreadsheet Phys. Med. Biol. 52 1909-22

Rogakou E P, Pilch D R, Orr A H, Ivanova V S and Bonner W M 1998 DNA double-stranded breaks induce histone H2AX phosphorylation on serine 139 J. Biol. Chem. $2735858-68$

Simon M, Barberet P, Delville M H, Moretto P and Seznec H 2011 Titanium dioxide nanoparticles induced intracellular calcium homeostasis modification in primary human keratinocytes. Towards an in vitro explanation of titanium dioxide nanoparticles toxicity Nanotoxicology 5 125-39

Snyder W S, Cook M J, Nasset E S, Karhausen L R, Parry Howell G P and Tipton I H 1975 Report of the task group on reference man ICRP publication 23 (Oxford: Pergamon)

Søyland C and Hasfjell S P 2000 A novel ${ }^{210} \mathrm{Po} \alpha$-particle irradiator for radiobiological experiments with retrospective $\alpha$-particle hit per cell determination Radiat. Environ. Biophys. 39 125-30

Szabó J, Fehér I, Pálfalvi J, Balásházy I, Dám A M, Polonyi I and Bagdándi E N 2002 In vitro cell irradiation systems based on ${ }^{210}$ Po alpha source: construction and characterisation Radiat. Meas. 35 575-8

Tisnek N, Kalaxhi E, Serkland C W and Irvesen J 2009 A 238Pu irradiator for exposure of cultured cells with alpha-radiation: construction, calibration and dosimetry Appl. Radiat. Isot. 67 1998-2002

Tung C J, Liu C S, Wang J P and Chang S L 2004 Calculations of cellular microdosimetry parameters for alpha particles and electrons Appl. Radiat. Isot. $61739-43$ 